NASA Contractor Report 4326

\title{
An Enhanced Integrated Aerodynamic Load/Dynamic Optimization Procedure for Helicopter Rotor Blades
}

Aditi Chattopadhyay and Y. Danny Chiu

CONTRACTS NAS1-18599 and NAS1-19000

OCTOBER 1990

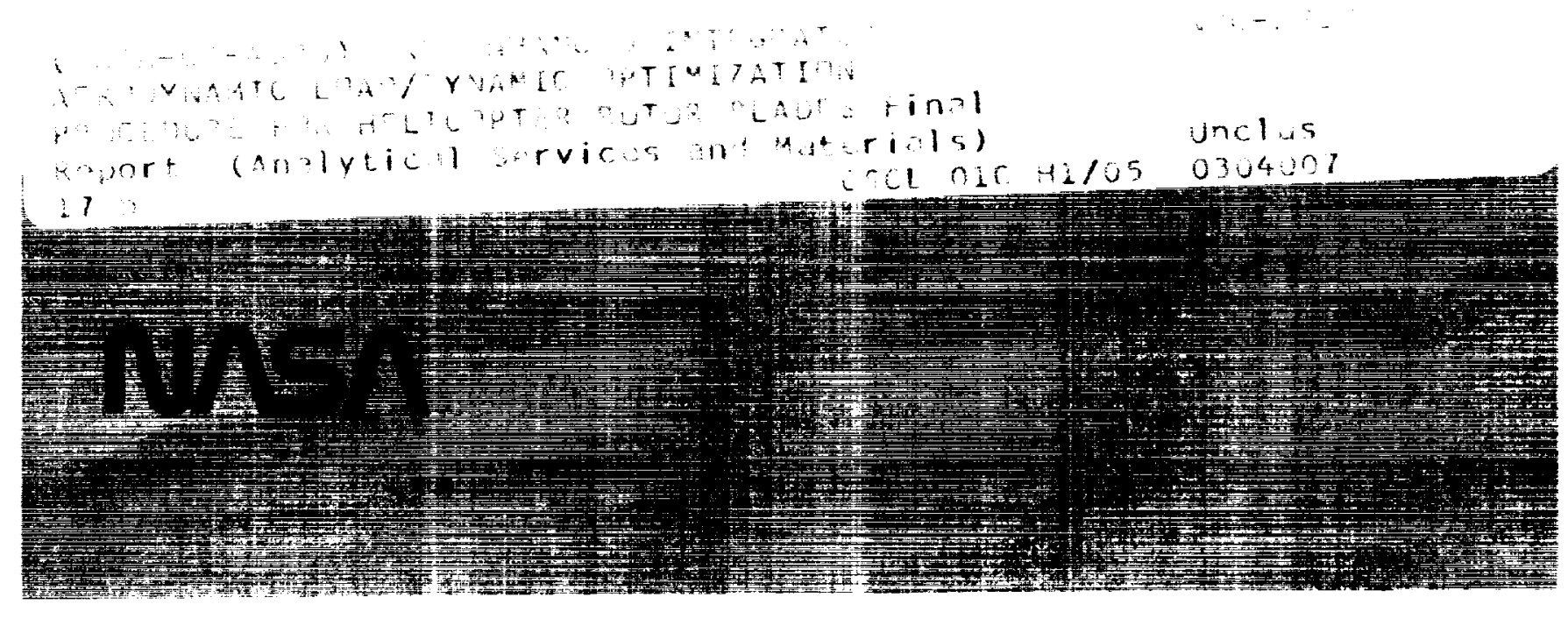




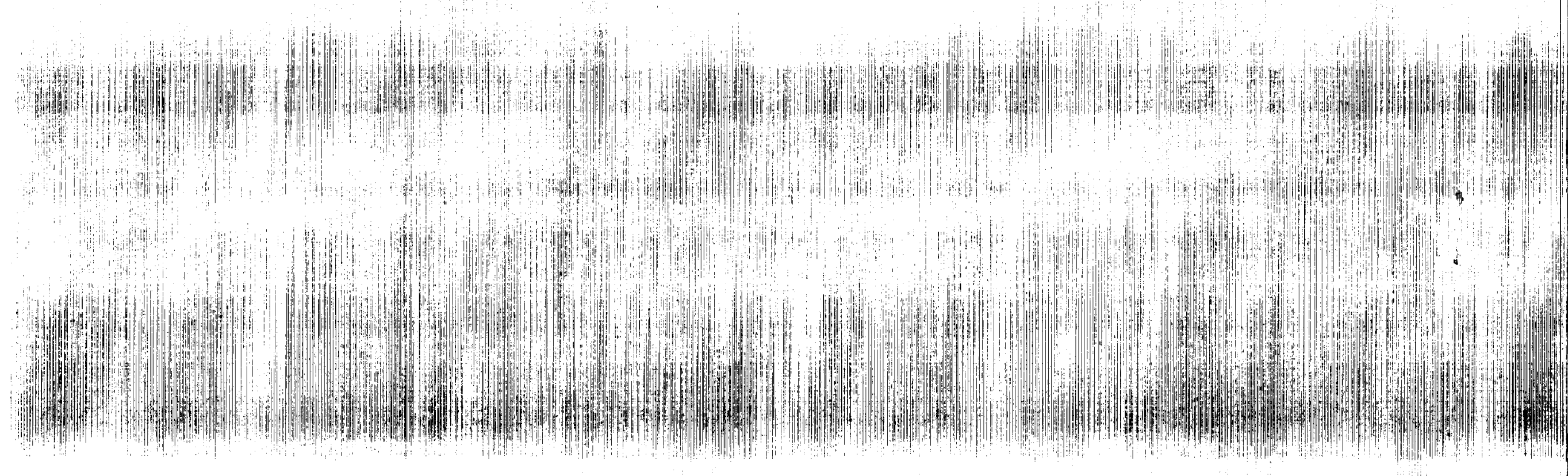

\section{3}

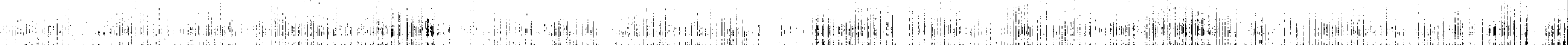
if 
NASA Contractor Report 4326

\title{
An Enhanced Integrated Aerodynamic Load/Dynamic Optimization Procedure for Helicopter Rotor Blades
}

\author{
Aditi Chattopadhyay \\ Analytical Services \& Materials, Inc. \\ Hampton, Virginia \\ Y. Danny Chiu \\ Lockheed Engineering \& Sciences Company \\ Hampton, Virginia
}

Prepared for

Langley Research Center

under Contracts NAS1 -18599 and NAS1-19000

\section{N/Sh}

National Aeronautics and

Space Administration

Office of Management

Scientific and Technical Information Division 


$$
\text { - }
$$




\title{
AN ENHANCED INTEGRATED AERODYNAMIC LOAD/DYNAMIC OPTIMIZATION PROCEDURE FOR HELICOPTER ROTOR BLADES
}

\author{
Aditi Chattopadhyay ${ }^{1}$ \\ Research Scientist \\ Analytical Services \& Materials, Inc. \\ Hampton, Virginia \\ Y. Danny Chiu ${ }^{2}$ \\ Research Engineer \\ Lockheed Engineering \& Sciences Company \\ Hampton, Virginia
}

\begin{abstract}
An enhanced integrated aerodynamic load/dynamic optimization procedure, developed for minimizing vibratory root shear forces and moments of a helicopter rotor blade is described. The optimization problem is formulated with $4 / \mathrm{rev}$ vertical and $3 / \mathrm{rev}$ inplane shears at the blade root as objective functions. Constraints are imposed on $3 / \mathrm{rev}$ radial shear, $3 / \mathrm{rev}$ flapping and torsional moments, 4/rev lagging moment, blade natural frequencies, weight, autorotational inertia, centrifugal stress and rotor thrust. The 'Global Criteria Approach' is used for formulating the multi-objective optimization. Design variables include spanwise distributions of blade bending stiffnesses, torsional stiffness, nonstructural mass, chord, radius of gyration and blade taper ratio. The program CAMRAD is coupled with an optimizer, which consists of the program CONMIN and an approximate analysis. The optimization procedure is applied to an advanced rotor as a reference design. Optimum blade designs, obtained with and without a constraint on the rotor thrust, are presented and are compared to the reference blade. Substantial reductions are obtained in the vibratory root forces and moments. As a byproduct, improvements are also found in some performance parameters which were not considered during the formulation of the optimization problem. The effect of thrust constraint on the values of the vibratory forces and moments is demonstrated by varying the magnitude of the prescribed thrust. A proper choice of the 'move limil' paremeter, used in the approximate analysis is shown to have significant effect on the optimum results.
\end{abstract}

\begin{tabular}{|c|c|}
\hline \multicolumn{2}{|r|}{ Notation } \\
\hline $\mathrm{AI}$ & autorotational inertia, $\mathrm{lb}-\mathrm{ft}^{2}$ \\
\hline$c$ & chord, $\mathrm{ft}$ \\
\hline$c_{r}$ & root chord, $\mathrm{ft}$ \\
\hline$c_{t}$ & tip chord, $\mathrm{ft}$ \\
\hline$C_{P}$ & power coefficient \\
\hline$C_{T}$ & thrust coefficient \\
\hline$C_{X}$ & propulsive force coefficient \\
\hline$E I_{x x} E I_{z 2}$ & bending stiffnesses, $\mathrm{lb}_{-} \mathrm{ft}^{2}$ \\
\hline$E I_{x x_{r}, E I_{z z_{r}}}$ & bending stiffnesses at blade root, $\mathrm{lb}-\mathrm{ft}^{2}$ \\
\hline$f_{3}, f_{4}, f_{5}, f_{6}$ & $\begin{array}{l}\text { natural frequencies of first four coupled } \\
\text { elastic modes, per rev }\end{array}$ \\
\hline$f_{r}$ & $3 /$ rev radial shear, $1 \mathrm{~b}$ \\
\hline$f_{x}$ & $3 / \mathrm{rev}$ inplane shear, $1 \mathrm{~b}$ \\
\hline$f_{z}$ & $4 / \mathrm{rev}$ vertical shear, lb \\
\hline$F, F$ & objective functions \\
\hline$\hat{F}$ & approximate objective function \\
\hline$F_{x}$ & inplane component of blade airload, $\mathrm{lb} / \mathrm{ft}$ \\
\hline$F_{2}$ & normal component of blade airload, $\mathrm{lb} / \mathrm{ft}$ \\
\hline $\begin{array}{l}g_{j} \\
\hat{g}\end{array}$ & $j^{\text {th }}$ constraint function \\
\hline GJ & torsional stiffness, $\mathrm{lb}_{-} \mathrm{ft}^{2}$ \\
\hline$G J_{r}$ & torsional stiffness at blade root, lb- $\mathrm{ft}^{2}$ \\
\hline$k_{r}$ & principle radius of gyration at blade root, $\mathrm{ft}$ \\
\hline$(L / D)_{\max }$ & maximum lift to drag ratio of an airfoil \\
\hline$m_{c}$ & 3/rev torsional moment, lb- $\mathrm{ft}$ \\
\hline$m_{x}$ & 3/rev flapping moment, lb-ft \\
\hline$m_{z}$ & 4/rev lagging moment, lb-ft \\
\hline ML & move limit \\
\hline $\mathrm{n}$ & integer, $0 \leq n \leq 1$ \\
\hline $\mathbf{N}$ & number of blade nodes \\
\hline
\end{tabular}

1 Member, AIAA, AHS.

2 Member, AHS. 


$\begin{array}{ll}\text { NCON } & \text { number of constraints } \\ \text { NDV } & \text { number of design variables } \\ \text { NSEG } & \text { number of blade segments } \\ \mathrm{R} & \text { blade radius, } \mathrm{ft} \\ s_{i} & \text { centrifugal stress in } i^{\text {th }} \text { segment, } \mathrm{lb} / \mathrm{ft}^{2} \\ s_{\max } & \text { maximum allowable stress, } \mathrm{lb} / \mathrm{ft}^{2} \\ \mathrm{~T} & \text { thrust, lb } \\ T_{r e f} & \text { thrust of reference rotor, } \mathrm{lb} \\ w_{j} & \text { nonstructural weight per unit length at } f^{\text {th }} \\ & \text { node, lb/ft } \\ \mathrm{W} & \text { total blade weight, lb } \\ \mathrm{x}, \mathrm{y}, \mathrm{z} & \text { reference axes } \\ \alpha & \text { angle of attack, deg } \\ \lambda & \text { taper ratio } \\ \varphi_{i} & i^{\text {th design variable }} \\ \mu & \text { advance ratio } \\ \sigma & \text { thrust-weighted solidity } \\ \Psi & \text { blade azimuth angle, deg } \\ \Omega & \text { rotor angular velocity, rad } / \mathrm{sec}\end{array}$

\section{Introduction}

Vibration has been a major source of problems in helicopters and its alleviation plays an important role in the rotor blade design process. Due to more stringent requirements in the permissible vibration levels and requirements for increased reliability, the helicopter industry is exploring methods to reduce vibration. In the past, conventional design methods mainly used the designer's experience and trial and error methods. Using these methods, rotor vibratory responses are reduced through post design addition of tuning masses, which incorporate weight penalty. Today with efficient optimization schemes available and improved helicopter analysis, attempts are being made to apply optimization techniques, at early stages of design, to the vibration problem.

Due to the importance of the problem, there has been a considerable amount of research aimed at reducing vibration using optimization, primarily at the blade level, as shown in Refs. 1- 11. In most of this work, the vibration is controlled by reducing the oscillatory hub shears and moments. In one such effort, the mode shape is changed to make it orthogonal to the aerodynamic forcing function and thereby reduce vibration ${ }^{1}$. An early attempt at optimum blade design for reduced vibration is due to Peters et al. ${ }^{2}$ where the objective was to place the natural frequencies away from the critical frequencies and thereby lower the hub shear. Refs. 3 and 4 present more recent developments in optimum blade designs for minimum weight with constraints on multiple natural frequencies, blade stress and autorotational inertia for articulated rotor blades with both rectangular and tapered planforms. However, the blade was assumed to be in vacuum and therefore the optimization was performed without airloads. The results of the above research indicate that by appropriately selecting the blade inertial, structural and sometimes aerodynamic characteristics, it is possible to minimize blade vibration.

In most of the previous work dealing with optimum blade designs for reduced vibration, the aerodynamic loads on the blade were prescribed and the effects of the design changes, during optimization, on the blade airloads were not included. In Ref. 10, for the first time, the integration of aerodynamic loads and dynamics was addressed by coupling a comprehensive helicopter analysis code, CAMRAD ${ }^{11}$, the nonlinear optimization algorithm, $\mathrm{CONMIN}^{12}$, and an approximate analysis technique. A combination of the blade root $4 /$ rev vertical shear and the blade weight was minimized with constraints on coupled elastic flap/lag natural frequencies, blade autorotational inertia and centrifugal stress. The use of the program CAMRAD permitted the design of the blade with calculated airloads and its presence in the closed-loop optimization procedure allowed the inclusion of the effects due to changes in these airloads with changes in design variables. The paper demonstrated significant reductions in the $4 / \mathrm{rev}$ vertical shear and blade weight, which were objective functions. As a byproduct, it was shown that optimization also reduced the total power loading required.

The research reported in Ref. 15 extends the work of Ref. 10 by including other sources of blade vibration that are transmitted to the hub. The purpose of this paper is to include details which were not included in Ref. 15 due to page limitations. In Ref. 15 a more comprehensive formulation was presented by including the $3 / \mathrm{rev}$ inplane and radial shears, the $3 / \mathrm{rev}$ flapping and torsional moments and the 4/rev lagging moment in the optimization formulation in the form of objective functions and/or constraints. In Ref. 10, the optimum rotor was required to maintain the same $C_{T} / \sigma$ and $C_{X} / \sigma$ as the reference rotor through a trim procedure, $\mathrm{C}_{\mathrm{T}}$ being the rotor thrust coefficient, $\mathrm{CX}$ the propulsive force coefficient and $\sigma$ the thrust weighted solidity of the blade. This allowed the thrust of the optimum rotor to be lower than that of the reference rotor due to the smaller solidity of the optimum blade. To avoid this loss in the rotor thrust, in Ref. 15, an additional constraint is imposed on the total thrust.

\section{Problem Description}

Optimization techniques are applied to minimize the vibratory blade loads of a four-bladed helicopter in forward flight. This is done by including the major sources of vibration (forces and moments) in the rotating frame that are transmitted to the nonrotating system as $4 / \mathrm{rev}$ harmonics of the longitudinal, lateral and vertical forces 
and the pitch, roll and yaw moments. For instance, in a four-bladed rotor, the $4 / \mathrm{rev}$ vertical shear at the blade root is transmitted as a 4/rev vertical force at rotor hub and the 3 and the 5/rev components of the inplane and the radial shears are transmitted as 4/rev forces in both lateral and longitudinal directions at the hub. Similarly, the 3 and the $5 / \mathrm{rev}$ components of the flapping and the torsional moments at the blade root affect the rotor hub in the form of oscillatory $4 / \mathrm{rev}$ pitch and roll moments. Finally the 4/rev lagging moment at the root causes oscillatory 4/rev yawing moment at the hub. Therefore, in this study, all of the 3 and the 4/rev components of these critical shear forces and moments are included in the optimization formulation in the objective functions and/or constraints. Although the $5 / \mathrm{rev}$ components of the inplane shear, the flapping and the torsional moments are important, their values are much smaller and therefore are not included. The excessive addition of weight, associated with most vibration reduction processes, is avoided by incorporating an upper limit constraint on the blade weight.

\section{Optimization Formulation}

The objective functions are the $4 /$ rev vertical shear, $f_{z}$, and the $3 /$ rev inplane shear, $f_{x}$, at the blade root. The following constraints are imposed: 1) upper and lower bounds on first four elastic coupled blade natural frequencies, $f_{3}, f_{4}, f_{5}$ and $f_{6}, 2$ ) lower bound on blade autorotational inertia, AI, 3) upper bound on total blade weight, $W, 4)$ upper bound on centrifugal stress on each blade segment, $\mathrm{si}_{\mathrm{i}} \mathrm{i}=1,2, \ldots$ NSEG (NSEG is the number of blade segments), 5) upper bound on $3 /$ rev radial shear, $f_{r}$, 6) upper bound on $3 /$ rev flapping moment, $m_{x}, 7$ ) upper bound on 3/rev torsional moment, $m_{c}, 8$ ) upper bound on $4 /$ rev lagging moment, $m_{z}, 9$ ) lower bound on total thrust, $\mathrm{T}$ and side constraints on design variables to avoid impractical solutions. The bounds are selected from a baseline design which will hereafter be called the 'reference' blade. It is required that the optimum blade maintains a certain level of the reference blade thrust. This leads to a lower bound constraint on the total thrust, $T$, which is expressed as

$$
T \geq n T_{\text {ref }}(o \leq n \leq 1)
$$

where $T_{\text {ref }}$ is the reference blade thrust. A method called the "Global Criteria approach"13 is used to formulate the multiple objective optimization problem. Using this method, the optimum solution is obtained by minimizing a 'global criterion' defined by the sum of the squares of the relative deviations of the individual objective functions from their respective individual optimum values. The optimization problem reduces to minimizing the global objective function, $F(\varphi)$, where

$$
F(\varphi)=\left[\frac{f_{z}(\varphi)-f_{z}\left(\varphi_{1}^{*}\right)}{f_{z}\left(\varphi_{1}^{*}\right)}\right]^{2}+\left[\frac{f_{x}(\varphi)-f_{x}\left(\varphi_{2}^{*}\right)}{f_{x}\left(\varphi_{2}^{*}\right)}\right]^{2}
$$

subject to the complete set of constraints

$$
g_{j}(\varphi) \leq 0 \quad j=1,2, \ldots, N C O N
$$

and the side constraints. The design variable vector $\varphi_{1}{ }^{*}$ is obtained by minimizing the single objective function $f_{z}(\varphi)$ subject to the set of constraints $g(\varphi)$ and the design variable vector $\varphi_{2}{ }^{*}$ is obtained by minimizing $f_{x}(\varphi)$ subject to the same set of constraints. In the present case, due to the nonlinear nature of the above function the objective function in Eqn. 2 is modified as follows :

$$
F(\varphi)=\sqrt{F}(\varphi)
$$

The design variables are the blade stiffnesses at the root, $E I_{z x_{m}} E I_{z z_{r}}, G J_{r}$, the taper ratio, $\lambda$, the root chord, $c_{r}$, the radius of gyration at the blade root, $\mathrm{k}_{\mathrm{r}}$ and the nonstructural weights $w_{j}, j=1,2, \ldots, N$, where $N$ is the total number of blade nodes.

\section{Blade Model}

In this section the modeling assumptions that are used to relate the design variables to the blade structural properties like weight, autorotational inertia and centrifugal stress are described. A linear taper is assumed along the blade planform, as shown in Fig. 1 (to reduce the number of design variables) and the blade taper ratio is expressed as follows :

$$
\lambda=\frac{c_{r}}{c_{t}}
$$

where $c_{r}$ is the root chord and $c_{t}$ is the tip chord. It is assumed that the blade stiffnesses arise solely from the blade structural component and the contribution of the nonstructural masses, the skin and honeycomb are assumed to be negligible. The following radial distributions are used for the stiffnesses:

$$
E I_{x x}(y)=E I_{x x}\left[\frac{y}{R}\left(\frac{c(y)}{c_{r}}-1\right)+1\right]^{4}
$$

where $R$ is the blade radius and $c(y)$ is the chord distribution. For a linear taper

$$
c(y)=c_{r}\left[\frac{y}{R}\left(\frac{c(y)}{c_{r}}-1\right)\right]
$$


Similar distributions are used for $\mathrm{EI}_{\mathrm{zz}}$ and GJ. The link between the intermediate design variables and the blade cross sectional properties like area, weight, autorotational inertia and centrifugal stress are described in detail in Ref. 10.

\section{Analysis}

The program CAMRAD is used for both blade dynamic and aerodynamic analyses. The program CONMIN, along with an approximate analysis technique, is used for optimization.

\section{Dynamic Analysis}

In CAMRAD the blade response is computed using rotating, free-vibration modes, equivalent to a Galerkin analysis. Ten bending modes, out of which seven are flapping (one rigid and six elastic) and three are lead-lag (one rigid and two elastic) and one rigid body torsion mode, are calculated. Blade resonances up to eight per revolution are included and therefore eight harmonics of the rotor revolution are retained in the air loads calculation. The blade loads are generated using the isolated rotor model of CAMRAD.

\section{Aerodynamic Analysis}

CAMRAD is also used to predict the aerodynamic loading on the blade. Some assumptions made, while running the code, include: uniform inflow, yawed flow on the rotor, unsteady aerodynamics model and no dynamic stall. A trim analysis is performed inside CAMRAD at each step of design optimization. Since the reference blade is a wind tunnel blade model, one of the wind tunnel options was selected for trimming the blade. The blade was trimmed to maintain the same $C_{T} / \sigma, C_{x} / \sigma$ and flapping angles as the reference blade using the collective and the cyclic (lateral and longitudinal) pitch and the shaft angle.

\section{Optimization Implementation}

The basic algorithm used is the method of Feasible Directions as implemented in the optimization program CONMIN. Since the optimization process requires many evaluations of the objective function and constraints before an optimum design is obtained, the process can be very expensive if full analyses are made for each function evaluation. The objective function and constraints are therefore approximated by linear Taylor series expansions based on the design variable values from CONMIN and the sensitivity information from the full analysis. Since the objective function and the constraints are all linearized, the optimization problem essentially reduces to a sequential linear programming (SLP) procedure. Specifically, if the objective function $F$, the constraint $g$, and their respective derivatives are calculated for the design variable $\varphi_{x}$ using an exact analysis, their values for an increment in the design variable $\Delta \varphi_{k}$ are as follows:

$$
\hat{F}=F+\sum_{k=1}^{N D V} \frac{\Delta F}{\Delta \varphi_{k}} \Delta \varphi_{k}
$$

$$
\begin{aligned}
& \text { and } \\
& \hat{g}=g+\sum_{k=1}^{N D V} \frac{\Delta g}{\Delta \varphi_{k}} \Delta \varphi_{k}
\end{aligned}
$$

where the quantities denoted $(\wedge)$ represent approximate values and NDV denotes the number of design variables. The assumption of linearity is valid over small increments in the design variable values and does not introduce large errors. A 'move limit' (ML), defined as the maximum fractional change of each design variable value, has been imposed as upper and lower bounds on each design variable $\varphi_{x}$. In this paper, equal values are used for boch upper and lower bounds.

\section{Test Problem}

The reference blade chosen for this work is a modification of a wind tunnel model of an advanced articulated rotor blade of the Growth Utility Rotor ${ }^{14}$. The modifications were made to the model to make it more suitabie for optimization studies. For example, a single airfoil is used throughout the blade span instead of the three different airfoils present in the advanced blade. Also, a rectangular planform is assumed for the reference blade rather than the tapered. The reference blade has a radius, $R=4.685 \mathrm{ft}$, rotational speed, $\Omega=639.5 \mathrm{rad} / \mathrm{sec}$, flap hinge offset value (normalized with respect to the radius) of 0.0534 and maximum linear twist of -16 degrees. Further details of the blade model can be found in Ref. 10. Optimum designs are obtained for a forward flight case with an advance ratio, $\mu=0.3$.

\section{Results}

In this section, results obtained by using the optimization procedure are presented. Optimum designs are obtained in 10-15 cycles. Oprimum blade designs are obtained with a thrust constraint of $T \geq T_{\text {ref }}$, i.e., the optimized blade must maintain at least the reference blade thrust $\left(n=1.0\right.$ in Eqn. 1). Note that when $T=T_{\text {ref, the }}$ solidities of the optimum and the reference blades will be the same since $\mathrm{C}_{T} / \sigma$ and $\mathrm{CX}_{\mathrm{X}} / \sigma$ are maintained constant through the trim procedure. Results are also obtained without the thrust constraint. This represents a case where the thrust can be reduced if $\sigma$ is reduced, as long 
as the quantities $\mathrm{C}_{T} / \sigma$ and $\mathrm{CX} / \sigma$ are maintained constant (in this case through the trim procedure), i.e., the thrust and the propulsive force per unit area are held constant during the optimization. The remaining constraints are the same for both cases. In addition results are also presented with varying magnitude of the required thrust, i.e., for different values of $n$ in Eqn. 1. For ease of reference, the following notation will be used

\section{Case 1: optimum design with thrust constraint, $T \geq T_{\text {ref }}$ \\ Case 2: optimum design without thrust constraint.}

Clearly Case 1 is of much more practical interest. However, Case 2 is included because it brings out some interesting phenomenological effects which illustrate important aspects of aerodynamic/dynamic interaction in rotorcraf. These integrated aspects are important to predict and understand in future efforts in integrated rotorcraft analysis and optimization.

\section{Optimization Results}

Table 1 presents a summary of the reference and the optimum designs in terms of the objective functions and constraints. By definition, a constraint is active when its value reaches one of the prescribed bounds after optimization. From Table 1, $\mathrm{f}_{6}$ (1st elastic lead-lag) is an active constraint in Case 1 . The $3 / \mathrm{rev} \mathrm{m}_{\mathrm{x}}$ and $\mathrm{m}_{\mathrm{c}}$ (flapping and torsional moments, respectively) and the thrust constraint are also active in Case 1 . The remaining constraints stay within or below the prescribed limits. There is a significant reduction ( 11.7 percent) in blade weight, from that of the reference blade, in Case 2. Fig. 2 shows that the centrifugal stresses in each segment remain below the prescribed upper bound, $s_{\max }$, and less than reference blade stresses for both Cases 1 and 2 .

Table 2 presents a summary of the reference and the optimized blade design variables (except for the nonstructural masses which are presented in Fig. 3). For Case 1 all of these design variables except $\mathrm{k}_{\mathrm{r}}$ remain unchanged, from the reference to the optimum. The optimum blade therefore remains rectangular in planform. Considerable changes, however, occur in Case 2 . The increases in $E I_{x x_{r}}$ and $E I_{z 2}$, from the reference blade values, in Case 2, are caused by the fact that the optimum blade is tapered, with $\lambda=1.24$, whereas the reference blade has a rectangular planform. Therefore, to account for the loss of stiffnesses towards the blade tip and to satisfy the frequency constraints, the stiffness values must increase at the root. It is interesting to note from Table 2 that the value of the design variable $\mathrm{GJ}_{\mathrm{r}}$ remains unchanged after optimization although the $3 / \mathrm{rev}$ torsional moment is constrained. This is because only a rigid body torsion mode is included in the analysis. In Case 2 there is a significant reduction in the blade root chord ( 37.8 percent) resulting in a reduced blade solidity ( 46.7 percent) from the reference blade value; the optimum blade in Case 2 with a smaller solidity permits less thrust to achieve the same $\mathrm{C}_{\mathrm{T}} / \sigma$ goal (Table 1).

The nonstructural weight distributions of the reference and the optimized blades are presented in Fig. 3. Reductions are shown in these weights, from reference to optimum, primarily over the blade inboard region. The weights increase towards the blade tip due to the autorotational inertia constraint. The inboard reductions of these weights are less in Case 1 than in Case 2. Table 2 and Fig. 4 present a comparison of the amplitudes of the $4 / \mathrm{rev}$ vertical and the $3 / \mathrm{rev}$ inplane shears, which are the objective functions. The $4 / \mathrm{rev}$ vertical hub shear is reduced by 10.9 percent and the $3 / \mathrm{rev}$ inplane shear is reduced by 4.1 percent, from the reference blade values, in Case 1 . The reductions are naturally larger in Case $2,89.8$ percent and 55 percent reductions in the vertical and inplane, respective!y. This is due to the fact that this rotor has much lower thrust.

It was of interest to investigate how much of the reduction in the vibratory forces and moments were caused by reductions in the blade section airloads. Therefore, the normal and the inplane components of the total aerodynamic forces on the section, resolved with respect to the hub plane, and denoted $F_{z}$ and $F_{x}$ respectively are plotted in Figs. $5-12$. Figure 5 presents the radial distribution and Figs. $6-8$ present the azimuthal distributions of $F_{z}$. These figures show that, for Case 2, optimization significantly reduces the amplitude of $F_{z}$ distribution of the advancing blade along the blade radius (Fig. 5). The amplitudes of the same distribution around the azimuth, plotted at three critical radial stations, $25 \%$ radius ( $y / R=0.25$; Fig. 6 ), the thrust-weighted equivalent chord point $(y / R=0.75$; Fig. 7) and blade tip $(y / R=0.99$; Fig. 8), for Case 2, are also reduced. No visible changes are observed in the above distributions for Case 1. The large reductions in the amplitude of $F_{z}$ distribution in Case 2 can be attributed to the lower thrust carried by the blade. Therefore, the larger reduction in the $4 / \mathrm{rev}$ vertical shear in Case 2 is brought about largely by the reduction in $\mathrm{F}_{\mathrm{z}}$. Fig. 9 presents the radial distribution and Figs. 10 - 12 present the azimuthal distributions of $F_{x}$ (at the same three radial locations as in $F_{z}$. Only Case 2 shows reductions in the amplitudes of $F_{x}$ distributions. The reason is that, in Case 2, the blade is being trimmed to produce the same $C_{T} / \sigma$ and $C_{X} / \sigma$ as the reference blade and no constraint is imposed on the thrust and the propulsive force. The smaller rotor eventually carries both less thrust and less propulsive force. Therefore, $F_{x}$ having a component of the thrust as well as the propulsive force embedded in it reduces significantly. This study clearly shows that in 
Case 2 the vibratory root shears and moments are reduced partly by proper blade tuning (through nonstructural weights) and frequency placements, but largely due to the reductions in the blade airloads. The mechanism is different in Case 1, where blade aerodynamic consideration is strongly coupled through the thrust constraint. In this case, the mean loads remain unchanged, however, the vibratory forces are reduced mainly through proper frequency placements and blade tuning.

\section{Effect on Thrust Constraint}

Since the thrust constraint was found to play an important role in the optimum blade design, it was of interest to study its role in greater details. This is done by varying the magnitude of the prescribed thrust (by changing the value of ' $n$ ', Eqn. 4) and comparing the forces and moments at the blade root. The results of this study are shown in Figs. 13 and 14. Figure 13 presents the variations of the root shear forces and Fig. 14 presents the variations of the root moments with changes in the thrust constraint. It is interesting to note, from these figures, that the thrust constraint does not affect the values of the forces and moments for values of $T \leq 0.54 T_{\text {ref. }}$. The magnitudes of the shear forces and moments increase with the increase in the magnitude of the prescribed thrust, as expected, above that value. For example there is a nearlinear increase in the values of the shear forces as indicated by Fig. 13. It is also of interest to note from Fig. 13 that the $3 / \mathrm{rev}$ inplane and radial shears vary almost identically with $\mathrm{n}$. Figure 14 shows gradual increases in the values of the $4 / \mathrm{rev}$ lagging and the $3 / \mathrm{rev}$ torsion moment above $n=0.54$. The increase in the $3 /$ rev flapping moment value tends to flatten out above $\mathrm{n}=0.90$. In both Figs. 13 and 14 the value of $n=1.0$ corresponds to the $100 \%$ thrust case (Case 1).

\section{Effect on Rotor Performance}

Although rotor performance was not considered while designing the optimum blades, it was of interest to investigate the effect of optimization on the performance. An important performance criterion is the total power required for a given task, which is a measure of economic efficiency. Therefore, it was of interest to see how it is affected. Recalling the fact that the optimum rotor in Case 2 is significantly smaller than the reference rotor, in Fig. 15 the power coefficient per unit solidity is compared. Fig. 15 indicates that the dynamic tuning did not increase the normalized power in Case 1. In Case 2 the solidity flexibility reduced the power as the thrust was reduced, however, the power loading (per unit solidiy) was more efficient for the optimum blade (9.2 percent lower than the reference blade) as shown in Fig. 15. This is further demonstrated by plotting the normalized section power coefficient (normalized with respect to its value at the tip of the advancing blade) against Mach number, at some typical radial locations, in Figs. $16-20$. These figures indicate that the general nature of the power distributions around the blade azimuth are similar for the reference and the optimum blades of both Cases 1 and 2. For example, at the thrust-weighted equivalent chord point (Fig. 18) the power is evenly distributed between the advancing and the retreating blades whereas towards blade tip $(y / R=$ 0.99; Fig. 20) most of the power is being used by the advancing blade. However, there is a definite reduction in the power requirement, from reference to optimum, as indicated by the reduced sizes of the diagrams. The reduction being more significant in Case 2. The reduction in the power is further investigated through a study of the blade angle of attack distributions. Figures $21-24$ show the angle of attack plotted against Mach number, at the same radial locations as the section power coefficient distribution. The $(\mathrm{L} / \mathrm{D})_{\max }$ shown in these figures, corresponds to the RC - 410 airfoils (Ref. 16) used in the reference blade. The figures indicate that the distributions are similar between the reference blade and the optimum blade in Case 1. However, for Case 2, both figures show that the advancing blade moves further away whereas the retreating blade moves closer to the $(\mathrm{L} / \mathrm{D})_{\max }$. This indicates that the retreating blade of the optimum rotor is operating more efficiently than the reference rotor, caused by a shift and increase in the mean section lift coefficient on the retreating blade after optimization. This explains the efficient power loading in the optimum blade for Case 2. A similar phenomenon is reported in Ref. 17 .

\section{Effect of Move Limit}

It was observed, during the optimization process using linear Taylor series approximation, that the move limits (ML) used in the approximate analyses of the objective functions and the constraints affected the optimum values to a great extent. Depending upon the value of ML, several different local minima resulted. One such occurrence is shown in Fig. 12, where the objective functions with a thrust constraint of $T \geq 0.75 T_{\text {ref }}(n=0.75$ in Eqn. 4) are plotted for different values of ML. The figure shows a large increase in the optimum value of the $4 /$ rev vertical shear, $f_{z}$, with a decrease in the value of ML from 0.1 to 0.02 . Similar changes are also in the optimum value of the $3 / \mathrm{rev}$ inplane shear, $f_{x}$. However, $f_{z}$ is found to be more sensitive to $\mathrm{ML}$ than $\mathrm{f}_{\mathrm{x}}$. For example, the optimum value of $\mathrm{f}_{\mathrm{z}}$ reduces by more than 25 percent as the value of $\mathrm{ML}$ is increased from 0.02 to 0.1 . The corresponding change is around 8 percent for $f_{x}$. This shows that the objective functions used are highly nonlinear with respect to the design variables with higher nonlinearity in case of the $4 / \mathrm{rev}$ vertical shear. Therefore, 
such nonlinear objective functions and constraints needed to be examined more carefully and a study was done on the most effective use of ML. In general, there is a tradeoff between a good local minimum and increased computer time. For instance, a small move limit provides a smoother convergence, but the rate of convergence is very slow and often the chances are that it does not converge to a good local minimum (e.g., the $f_{z}$ value in Fig. 25 with $\mathrm{ML}=0.02$ ). A large move limit gives a faster rate of convergence with more chances of oscillation (in case of a nonlinear function), often running into mathematical singularity (i. e., negative objective function). It was therefore decided to use a variable move limit procedure in which the optimization procedure is started with a large ML and the process continued until the approximation yields negative objective functions. At this point a switch is made to several sets of smaller values of ML. The ML value that yields the best local minimum is selected to be the optimum design. In the present analysis, move limits in the range of $0.1-0.01(10-1$ percent) have becn used.

\section{Concluding Remarks}

In this paper an integrated acrodynamic/dynamic optimization approach has been described for rotor blades to obtain reduced vibratory root loads. The $4 / \mathrm{rev}$ vertical shear and the 3/rev inplane shear forces are minimized for an advanced articulated rotor blade under forward flight conditions. The 'Global Criteria Approach' is used for formulating the multi-objective optimization problem. Constraints are imposed on the remaining vibratory loads - the $3 / \mathrm{rev}$ radial shear, the 3/rev flapping and torsional moments and the 4/rev lagging moment. Constraints are also imposed on blade natural frequencies, autorotational inertia, centrifugal stress and rotor thrust. The program CAMRAD is used for the acrodynamic and dynamic analysis of the blade and the program CONMIN, along with an approximate analysis technique, is used for the optimization. The blade is trimmed at each step of design optimization. The optimum designs are compared with a reference blade. Two optimum design cases were studied. In Case 1 the thrust of the optimum rotor was constrained to be the same as the reference rotor and in Case 2 no constraint was imposed on the thrust. The effect of thrust constraint was studied in detail. Rotor perfomance, a criterion, not included while designing the optimum blades, was also studied. The effect of move limit used in the approximate analysis on the optimum solution, was investigated.

Following are the conclusions that are made from this study:

1. The Global Criteria Approach is effective in solving the multiple objective problem The integrated op- timization scheme is very efficient and results are obtained in $10-15$ cycles.

2. The procedure yields significant reductions in the objective functions. The $4 / \mathrm{rev}$ vertical shear is reduced by 10.9 and 89.8 percent and the $3 /$ rev inplane shear is reduced by 4.1 and 55 percent in Cases 1 and 2, respectively. The reductions are larger in Case 2 due to the lower thrust of the rotor.

3. The amplitudes of the local section airloads (normal and inplane) are substantially reduced in Casc 2, proving it to be the driver behind the large reductions in the vibratory root forces and moments in this design. This airload reduction comes about along with a reduction in rotor thrust. In the practical application of a Case 1 deign, no reduction occurs in the airloads due to the thrust constraint and the vibratory forces and moments are reduced through blade tuning and frequency placement.

4. In Case 1 the optimum blade retains the same normalized power requirement as that of the reference blade and has a more efficient power loading than the reference blade for Case 2.

5. A proper choice of the move limit is important in arriving at a better local minimum.

\section{Acknowledgements}

This work was performed at the NASA Langley Research Center under NASA contracts NAS1- 18599 and NAS1-19000 for LE\&SC, technical monitor Dr. Howard M. Adelman. The authors greatly appreciate the uscful discussions with Mr. Wayne Mantay, Chief, Aeromechanics Division, Aerostructure Directorate, AVSCOM.

\section{References}

1. Taylor, R. B., "Helicopter Vibration Reduction by Rotor Blade Modal Shaping", Proc. of the 38th Annual Forum of the AHS, May 4-7, 1982, Anaheim, California.

2. Peters, D. A., Ko, Timothy, Rossow, Mark P., "Design of Helicopter Rotor Blades for Desired Placement of Natural Frequencics," Proc. of the 39th Annual Forum of the AHS, May 9-11, 1983, St. Louis, Missouri.

3. Chattopadhyay, Aditi and Walsh, Joanne L., "Minimum Weight Design of Rectangular and Tapered Helicopter Rotor Blades with Frequency Constraints," Proc. of the 2nd Int. Conference on Rotorcraft Basic Research, February 16-18, 1988, College Park, Maryland. Also available as NASA TM-100561, February 1988.

4. Chattopadhyay, Aditi and Walsh, Joanne L., "Minimum Weight Design of Rotorcraft Blades with Multiple Frequency and Stress Constraints," Proc. 
AIAA/ASME/ASCE/AHS 29th Structures, Structural Dynamics and Materials Conference, Williamsburg, Virginia, April 18-20, $1988 . \quad$ AIAA Paper No. 88-2337-CP. Also available as NASA TM-100569, March 1988.

5. Peters, D. A. Rossow, Mark P., Korn, Alfred, and Ko, Timothy, "Design of Helicopter Rotor Blades for Optimum Dynamic Characteristics," Computers \& Mathematics with Applications Vol 12A, No. 1, 1986, pp 85-109.

6. Hanagud, S., Chattopadhyay, Aditi, Yillikci, Y. K., Schrage, D., and Reichert, G., "Optimum Design of a Helicopter Rotor Blade," Paper No. 12, Proc. of the 12th European Rotorcraft Forum, September 2225, 1986, Garmisch-Partenkirchen, West Germany.

7. Celi, R. and Friedmann, P. P., "Efficient Structural Optimization of Rotor Blades with Straight and Swept Tips," Proc. of the 13th European Rotorcraft Forum, Arles, France, September 1987. Paper No. 3-1.

8. Lim, Joon, W. and Chopra, Inderjit, "Aeroelastic Optimization of a Helicopter Rotor," Proc. of the 44th Annual Forum of the AHS, June 16-18, 1988, Washington, D. C.

9. Weller, W. H. and Davis M. W., "Experimental Verification of Helicopter Blade Designs Optimized for Minimum Vibration," Proc. of the 44th Annual Forum of the AHS, June 16-18, 1988, Washington, D. C.

10. Chattopadhyay, Aditi, Walsh, Joanne L. and Riley, Michael F., "Integrated Aerodynamic/Dynamic Optimization of Helicopter Blades," Proc. AIAA/ASME/ASCE/AHS 30th Structures, Structural Dynamics and Materials Conference, Mobile,
Alabama, April 3-5, 1989. AIAA Paper No. 891269. Also available as NASA TM-101553, February, 1989.

11. Johnson, W., "A Comprehensive Analytical Model of Rotorcraft Aerodynamics and Dynamics," Part II: User's Manual, NASA TM 81183, June 1980.

12. Vanderplaats, G. N., "CONMIN - A Fortran Program for Constrained Function Minimization," User's Manual, NASA TMX-62282, August 1973.

13. Rao, S. S., "Multiobjective Optimization in Structural Design with Uncertain Parameters and Stochastic Processes," AIAA Journal, Vol. 22, No. 11, November 1984.

14. Yeager, W., Mantay, W., Wilbur, M., Cramer, R., and Singleton, J., "Wind-Tunnel Evaluation of an Advanced Main-Rotor Blade Design for a Utility Class Helicopter," NASA TM-89129, AVSCOM TM-87-B-8, September 1987.

15. Chattopadhyay, Aditi and Chiu, Y. Danny, "An Enhanced Integrated Aerodynamic Load/Dynamic Approach to Optimum Rotor Blade Design," Proc. of the 46th Annual Forum of the AHS, May 21-23, 1990, Washington, D. C.

16. Noonan, Kevin W., "Aerodynamic Characteristics of Two Rotorcraft Airfoils Designed for Application to the Inboard Region of a Main Rotor Blade," NASA TP-3009, AVSCOM TR-90-B-005, 1990.

17. Chattopadhyay, Aditi and Jones, Henry E., "Performance of an Optimized Rotor Blade at Orf-Design Flight Conditions," Proc. of the AHS and NASA Ames First Vertical Lift Aircraft Design Conference, San Francisco, CA, January 1990. 
Table 1 Contraint Values

\begin{tabular}{|c|c|c|c|c|c|c|}
\hline & & \multirow{2}{*}{$\begin{array}{c}\text { Reference } \\
\text { blade }\end{array}$} & \multicolumn{2}{|c|}{ Prescribed Bounds } & \multicolumn{2}{|c|}{ Optimum } \\
\hline & & & lower & upper & $($ Case 1) & $\left(\right.$ Case 2) ${ }^{b}$ \\
\hline$f_{3}$ (per rev) & (flap) & 3.07 & 3.05 & 3.50 & 3.13 & 3.31 \\
\hline$f_{4}$ (per rev) & (flap) & 6.76 & 6.50 & 6.90 & 6.87 & 6.90 \\
\hline$f_{5}$ (per rev) & (flap) & 9.28 & 9.25 & 9.50 & 9.38 & 9.25 \\
\hline$f_{6}$ (per rev) & (lead-lag) & 12.63 & 12.50 & 12.75 & 12.75 & 12.71 \\
\hline $\mathrm{AI}\left(\mathrm{lb}-\mathrm{ft}^{2}\right)$ & & 19.75 & 19.75 & - & 20.30 & 19.75 \\
\hline W (lb) & & 3.41 & - & 3.41 & 3.39 & 3.01 \\
\hline $3 /$ rev $f_{r}(l b)$ & & 2.81 & - & 2.81 & 2.75 & 1.08 \\
\hline $3 / \mathrm{rev} \mathrm{m}_{\mathrm{x}}(\mathrm{lb}-\mathrm{ft})$ & & 0.59 & - & 0.59 & 0.59 & 0.26 \\
\hline 3/rev me (lb-ft) & & 0.23 & - & 0.23 & 0.23 & 0.05 \\
\hline $4 /$ rev $m_{z}$ (lb-ft) & & 0.70 & - & 0.70 & 0.64 & 0.18 \\
\hline Thrust, $T$ (lb) & & 298.70 & $298.70^{c}$ & - & 298.70 & 158.50 \\
\hline
\end{tabular}

${ }^{2}$ Case 1 : optimum design with thrust constraint, $\mathrm{T} \geq \mathrm{T}_{\text {ref }}$

${ }^{b}$ Case 2 : optimum design without thrust constraint

${ }^{\mathrm{c}}$ For Case 1 only

Table 2 Optimiation results

\begin{tabular}{|c|c|c|c|}
\hline & $\begin{array}{l}\text { Reference } \\
\text { blade }\end{array}$ & \begin{tabular}{|l} 
Optimum \\
(Case 1) \\
\end{tabular} & \begin{tabular}{|l} 
Optimum \\
(Case 2) $^{\mathrm{b}}$ \\
\end{tabular} \\
\hline$E I_{\partial x_{r}}\left(b b-f t^{2}\right)$ & 10277.00 & 10277.00 & 10300.00 \\
\hline$E I_{z z_{r}}\left(l b-f t^{2}\right)$ & 354.00 & 354.00 & 378.00 \\
\hline$G J_{r}\left(b b-f t^{2}\right)$ & 261.00 & 261.00 & 261.00 \\
\hline$k_{r}(f t)$ & 0.27 & 0.16 & 0.17 \\
\hline$\lambda$ & 1.00 & 1.00 & 1.24 \\
\hline$c_{r}(f t)$ & 0.45 & 0.45 & 0.28 \\
\hline $4 / \operatorname{rev} \Upsilon_{z}(l b)$ & 0.16 & 0.14 & 0.016 \\
\hline $\begin{array}{l}\text { Percent reduc- } \\
\text { tion }\end{array}$ & - & 10.90 & 89.80 \\
\hline $3 /$ rev $f_{x}(l b)$ & 3.17 & 3.04 & 1.43 \\
\hline $\begin{array}{l}\text { Percent reduc. } \\
\text { tion }^{c}\end{array}$ & - & 4.10 & 55.00 \\
\hline
\end{tabular}

${ }^{a}$ Case 1: Optimum design with thrust constraint,

$T \geq T_{\text {ref }}$

${ }^{b}$ Case 2: Optimum design without thrust constraint

${ }^{c}$ From reference blade 


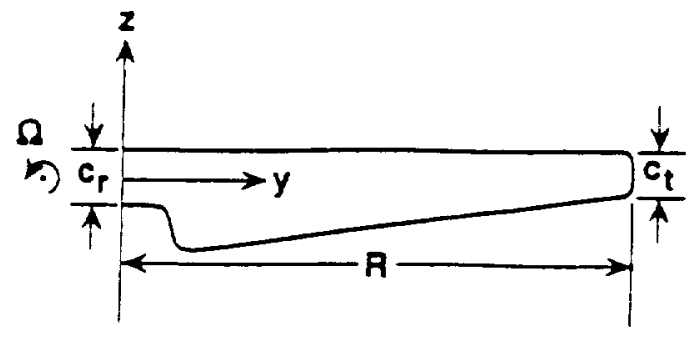

Fig. 1 Rotor blade model.

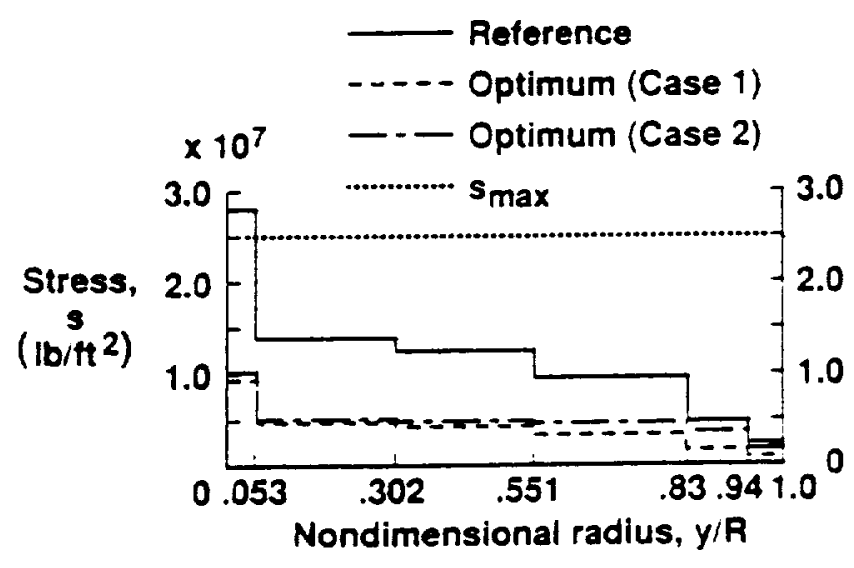

Fig. 2 Centrifugal stress distributions.

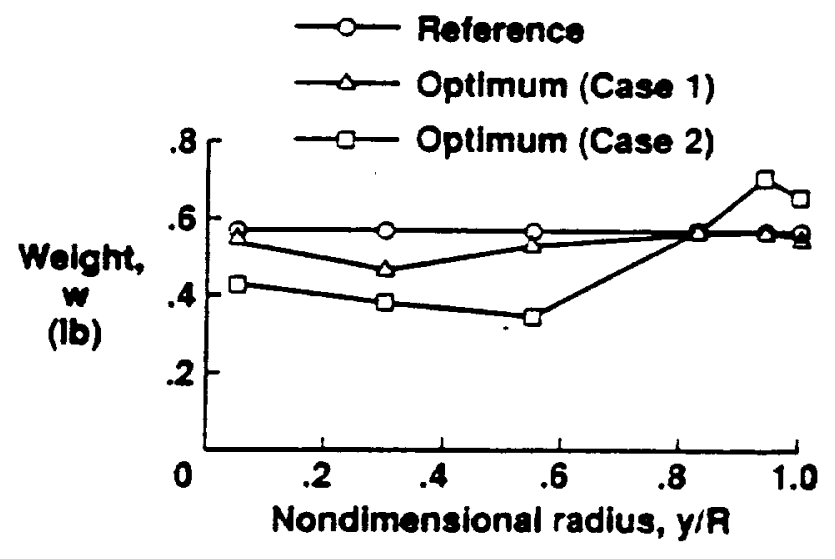

Fig. 3 Nonstrucaural weight disuributions.

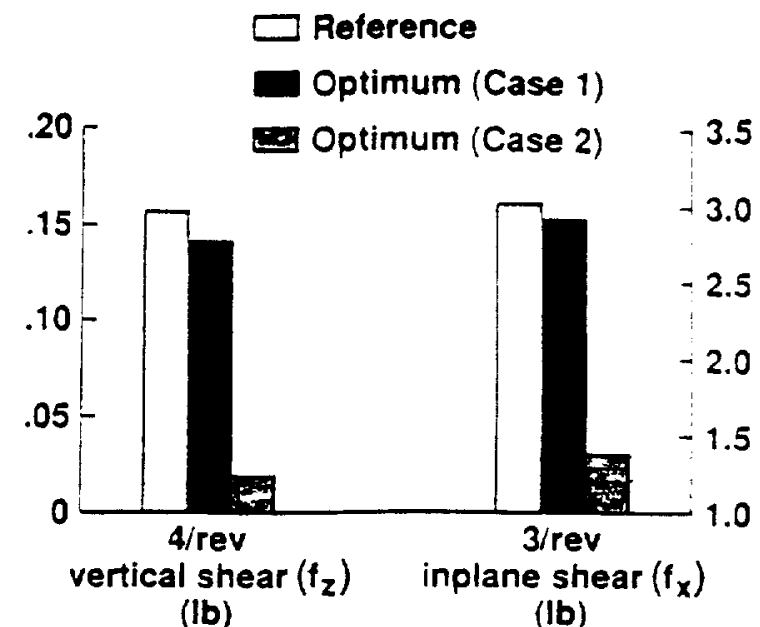

Fig. 4 Comparison of objective functions.

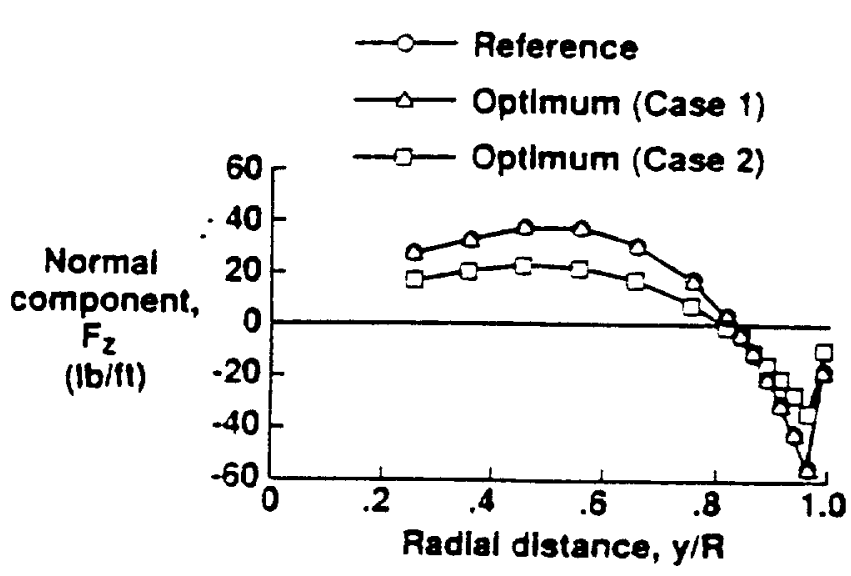

Fig. 5 Radial distributions of normal component of airlœad, $F_{z} ; \Psi=90^{\circ}, \mu=0.3$

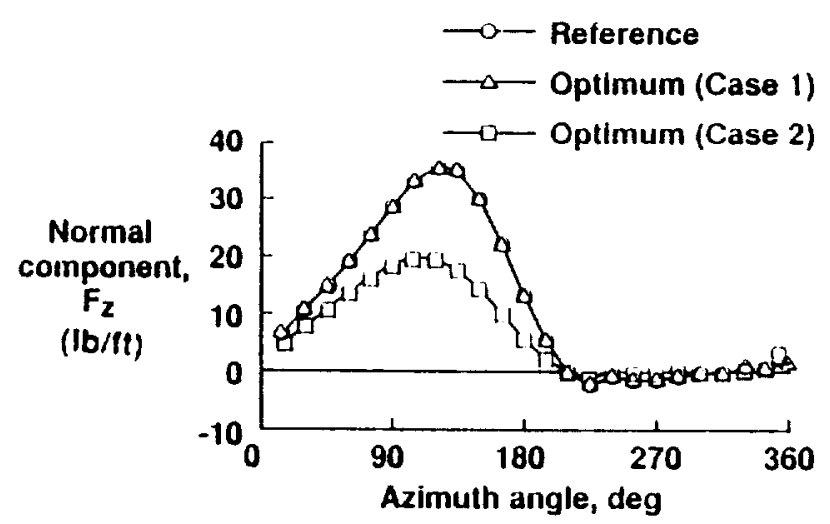

Fig. 6 Azimuthal distributions of normal component of airlcad , $F_{z i} y_{R}=0.25, \mu=0.3$. 


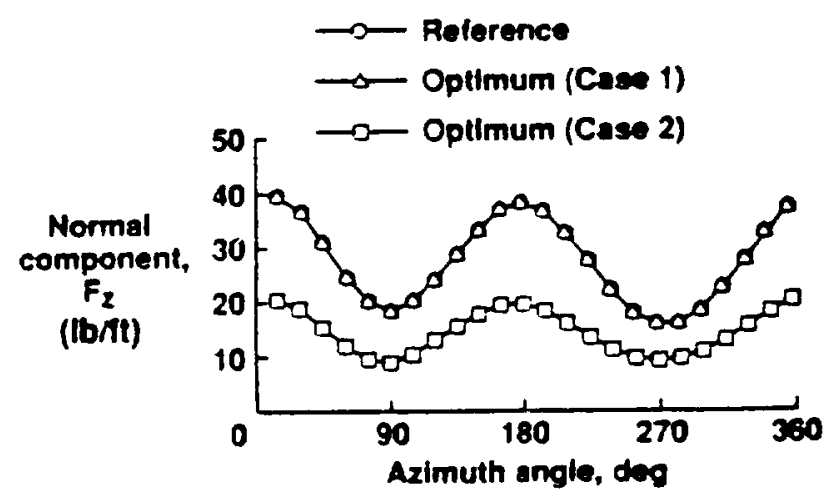

Fig. 7 Azimuthal distributions of normal component of airload, $F_{2} ; \not / R=0.75, \mu=0.3$.

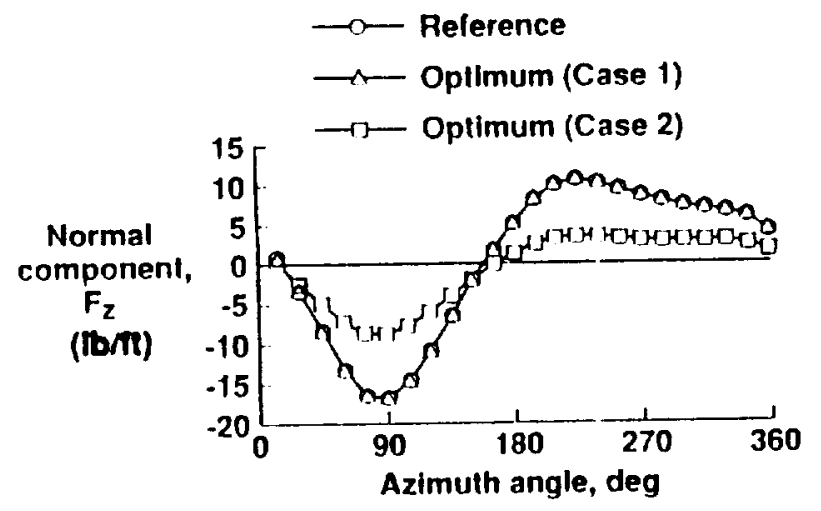

Fig. 8 Azimuthal distributions of normal component of airload, $F_{z} ;$ 牧 $=0.99, \mu=0.3$.

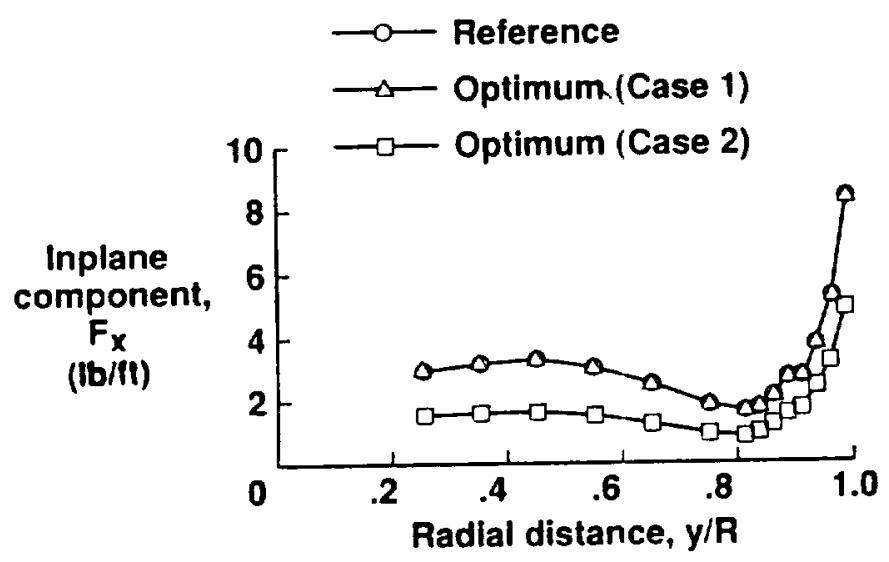

Fig. 9 Radial distributions of inplane component of airload, $F_{x} ; \Psi=90^{\circ}, \mu=0.3$.

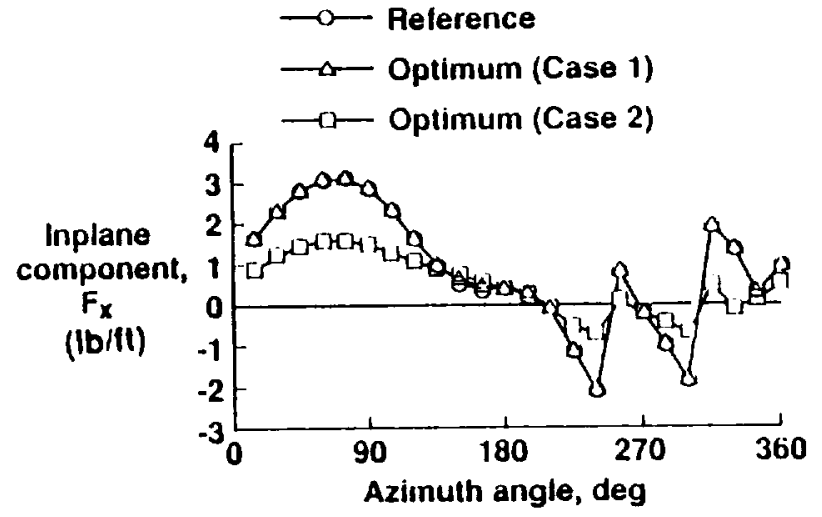

Fig. 10 Azimuthal distributions of inplane component of airload, $F_{x} ;$ 次 $=0.25, \mu=0.3$.

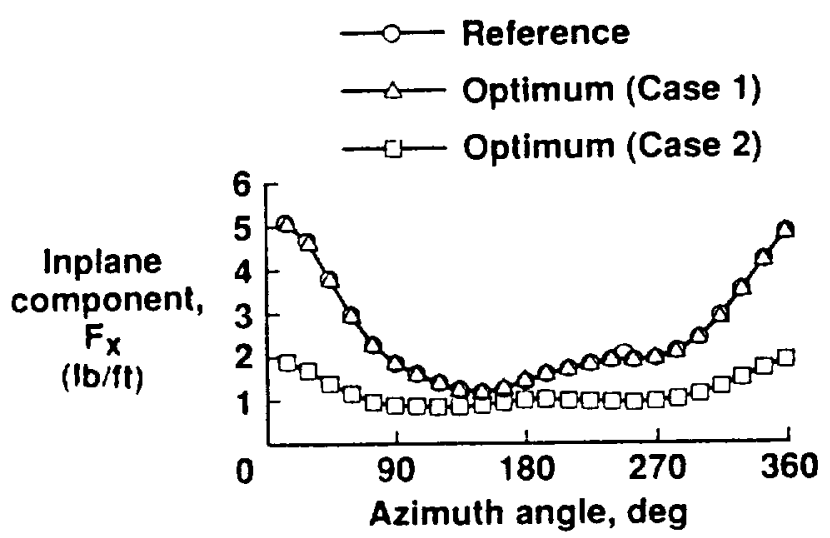

Fig. 11 Azimuthal distributions of inplane component of airload, $F_{x} ; y / R=0.75, \mu=0.3$.

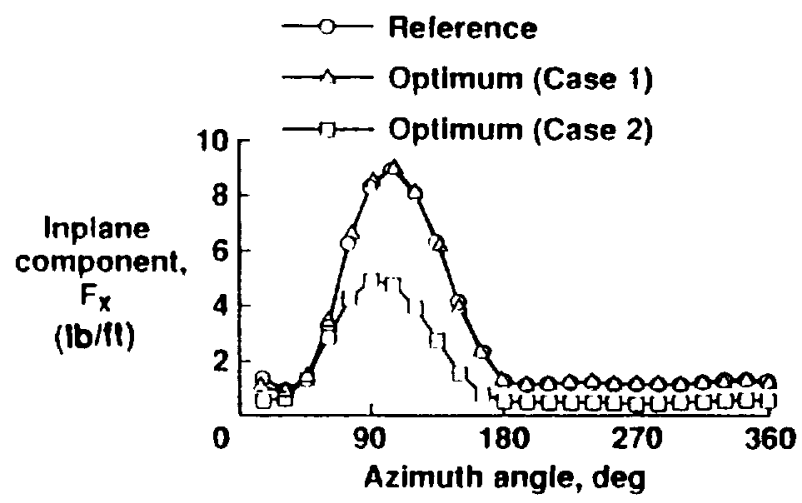

Fig. 12 Azimuthal distributions of inplane component of airload, $F_{x} ; \not / R=0.99, \mu=0.3$. 


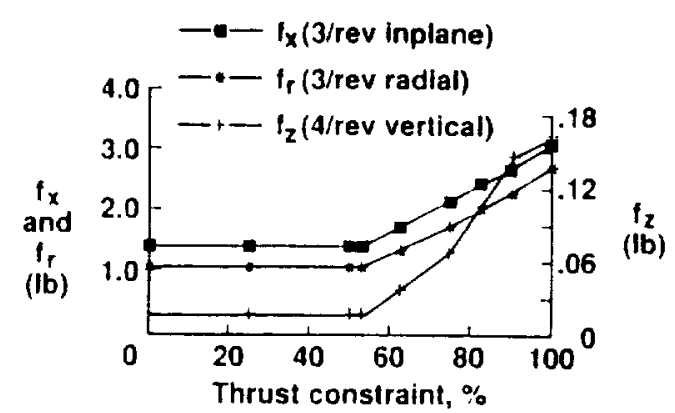

Fig. 13 Effect of thrust constraint on root shear forces.

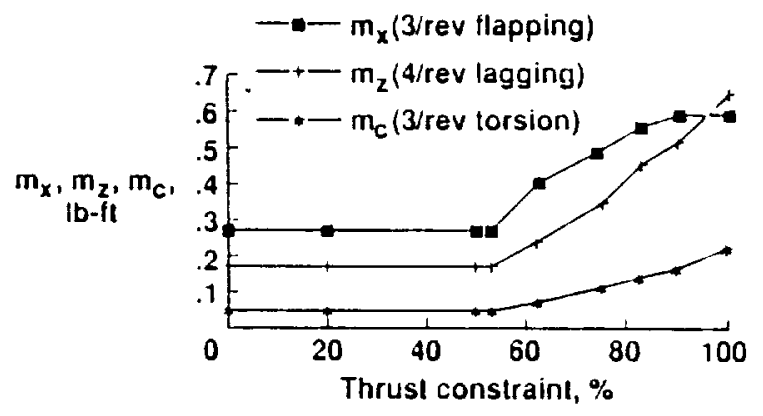

Fig. 14 Effect of thrust constraint on root moments.

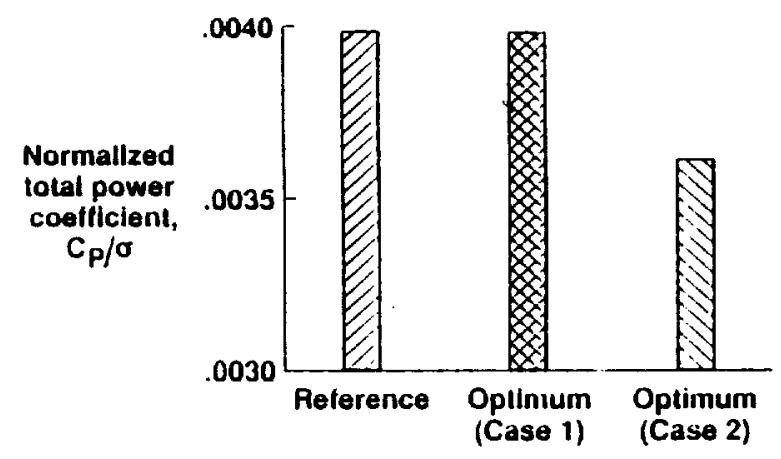

Fig. 15 Comparison of normalized total power coefficient.

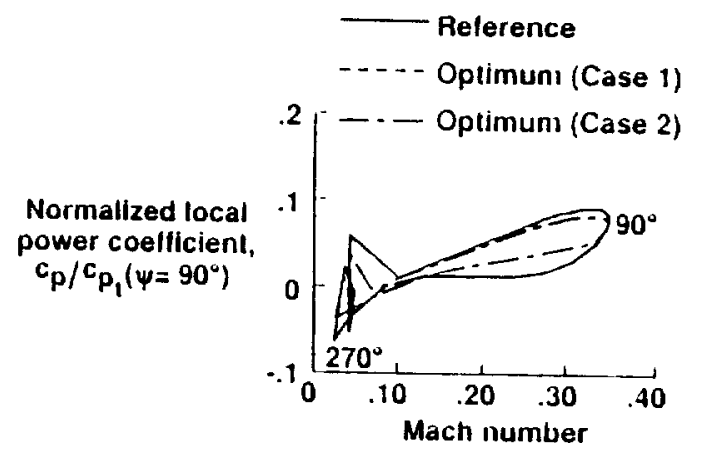

Fig. 16 Variation of normalized local power coefficient with Mach number; $Y_{R}=0.25$.

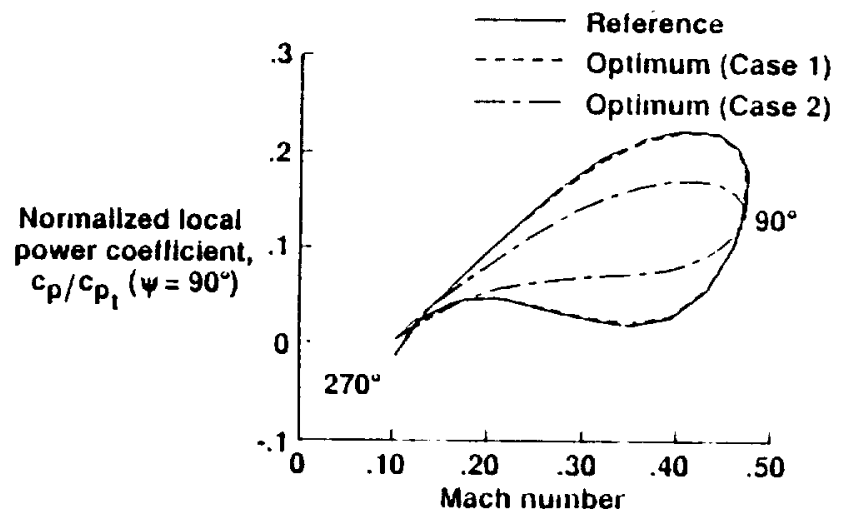

Fig. 17 Variation of normalized local power coefficient with Mach number; $x / R=0.45$.

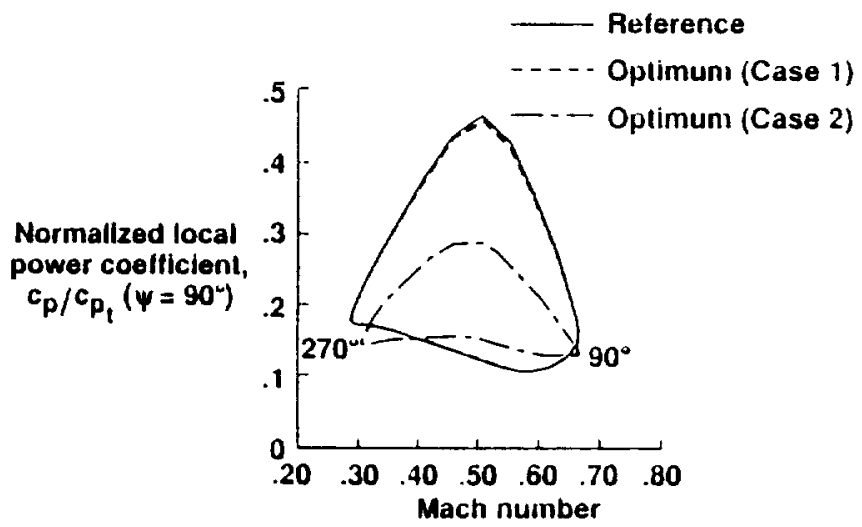

Fig. 18 Variation of normalized local power coefficient with Mach number; $y / R=0.75$. 


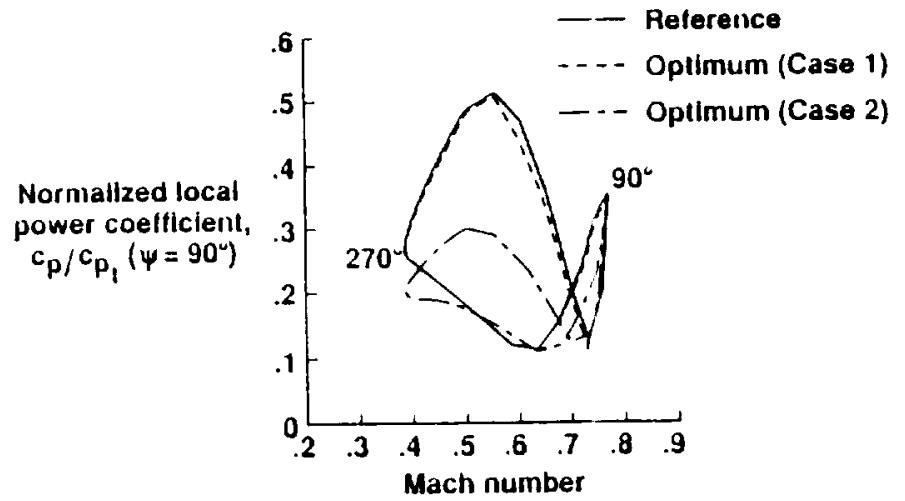

Fig. 19 Variation of normalized local power coefficient with Mach number; $y / R=0.91$.

- Reterence

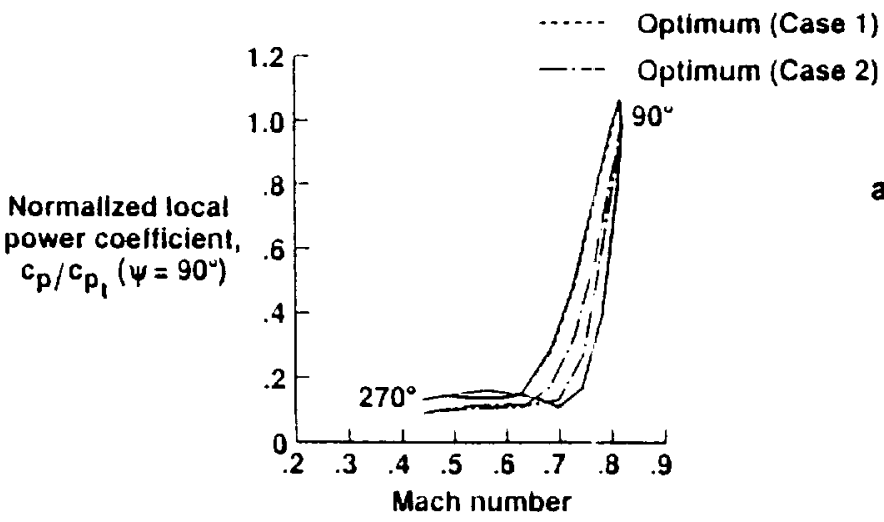

Fig. 20 Variation of normalized local power coefficient with Mach number; $y / R=0.99$

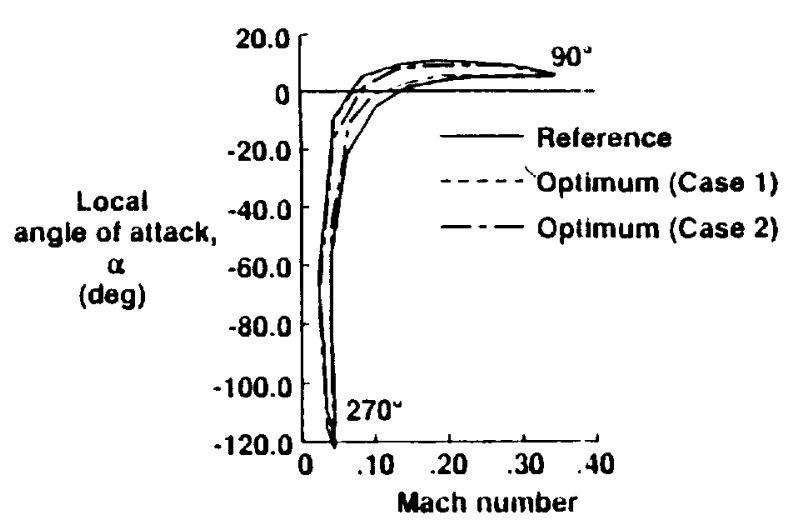

Fig. 21 Variation of local angle of attack with Mach number; $y / R=0.25$.

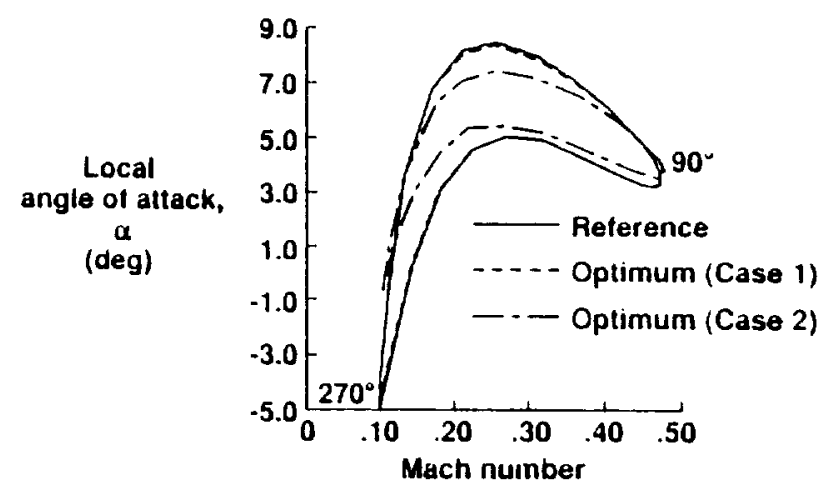

Fig. 22 Variation of local angle of attack with Mach number; $y / R=0.45$.

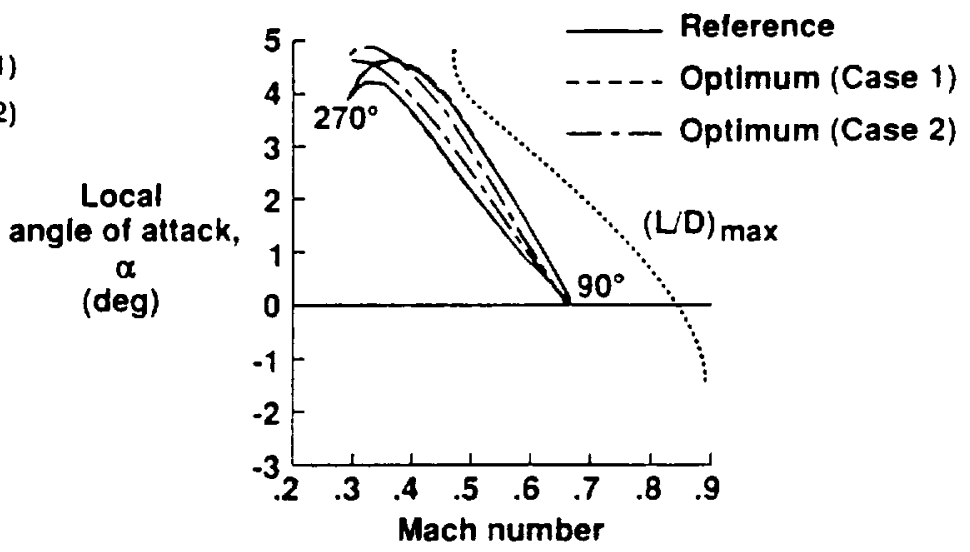

Fig. 23 Variation of local angle of attack with Mach number, $y / k=0.75$.

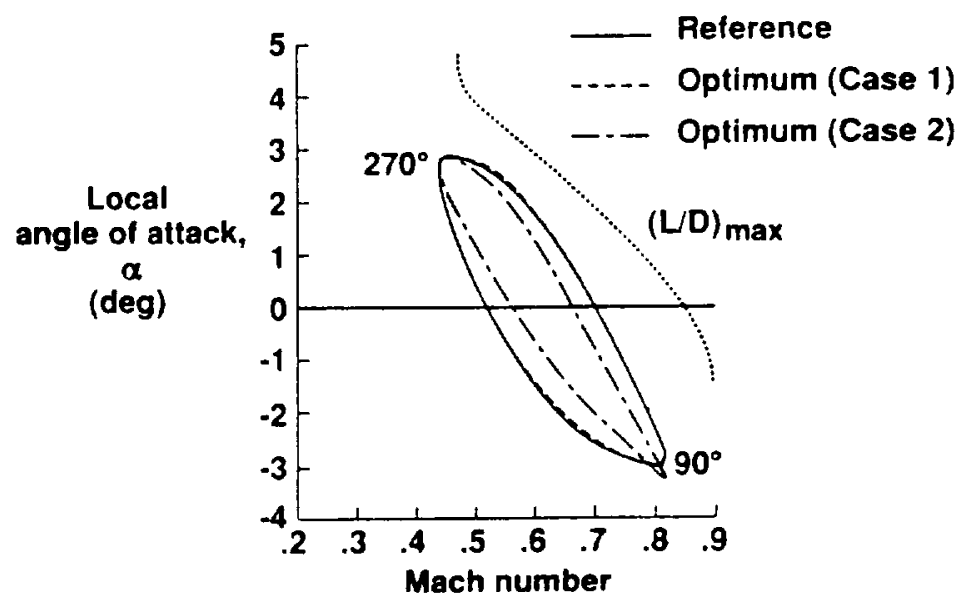

Fig. 24 Variation of local angle of attack with Mach number; $y / R=0.99$ 


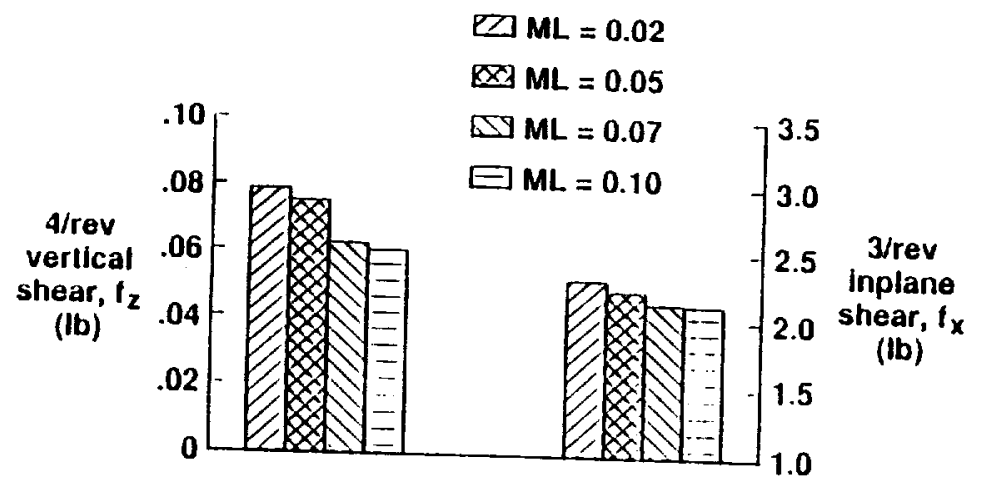

Fig. 25 Effect of move limit on objective functions. 




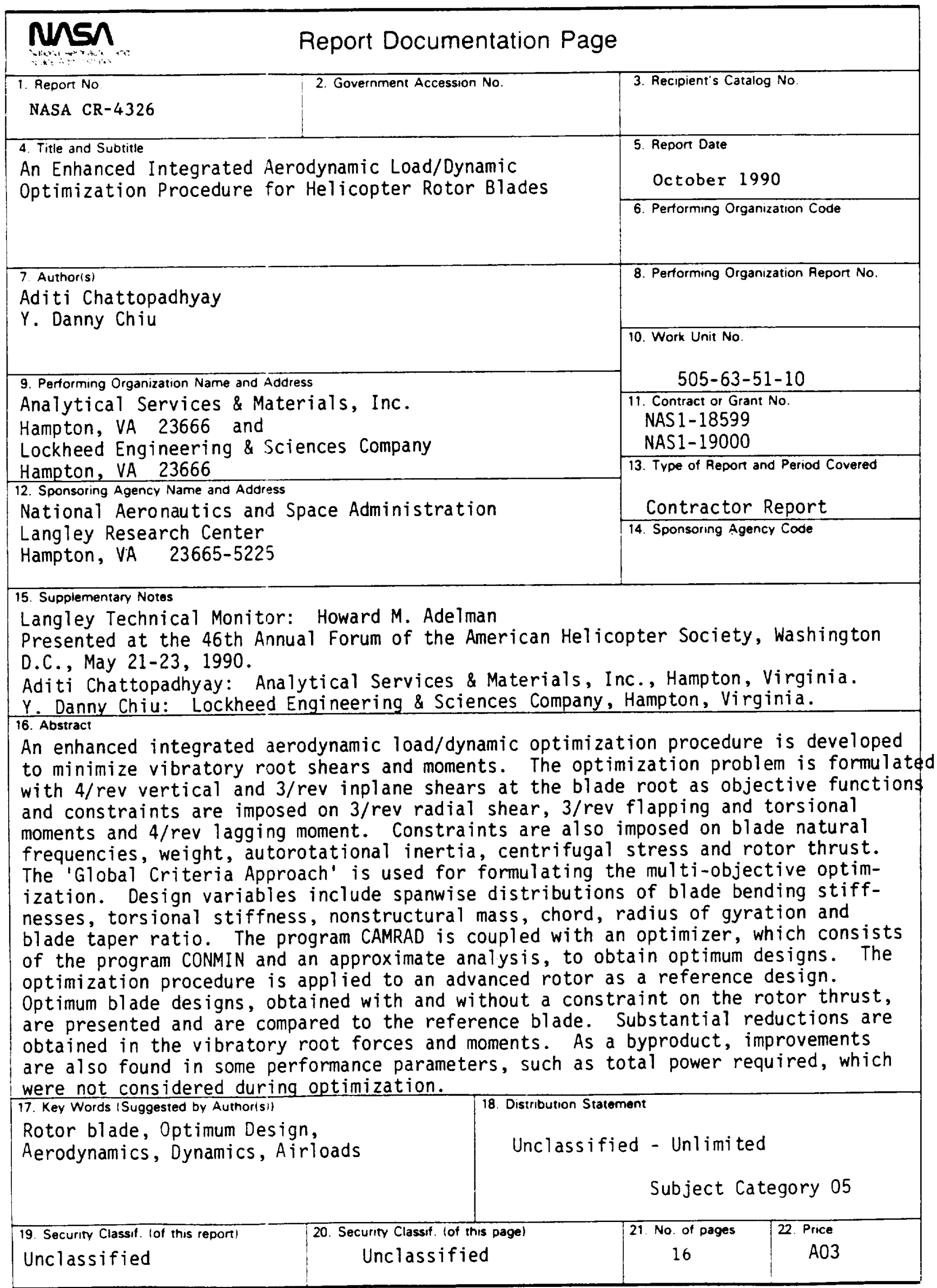


